Abstract 2 Table 1 Inclusion and exclusion criteria for HACX

\begin{tabular}{ll}
\hline Inclusion criteria & Symptoms suggestive of myocardial ischaemia \\
\hline & With ECG changes including: ST depression; T wave \\
inversion in V1-4; Dynamic T wave changes & OR positive troponin I assay \\
Exclusion criteria & Unexplained anaemia $(\mathrm{Hb}<10)$ \\
& Hypoxia \\
& Acute renal failure \\
& Loss of consciousness \\
& Recent trauma \\
& Overt sepsis \\
Immediate medical therapy includes & Aspirin $300 \mathrm{mg}$ \\
& Clopidogrel $600 \mathrm{mg}$ \\
& Fondaparinux $2.5 \mathrm{mg}$ \\
& Eptifibatide bolus $(180 \mathrm{mg} / \mathrm{kg})$ as long as no \\
bleeding contraindications
\end{tabular}

(42.4\%) of patients were treated with medical therapy alone. NSTEACS (encompassing NSTEMI and unstable angina) was the discharge diagnosis for $75.4 \%$ of HACX patients. $10 \%$ of patients had another cause for chest pain symptoms (including pericarditis and, myocarditis); $14.6 \%$ had a non-cardiac diagnosis. Mean time from presentation to angiography was pre-HAC-X 7349 mins ( \pm 6836 ) and post HAC-X 754 mins $( \pm 458)(p<0.0001)$ (see Abstract figure 1). Pre-HAC-X mean

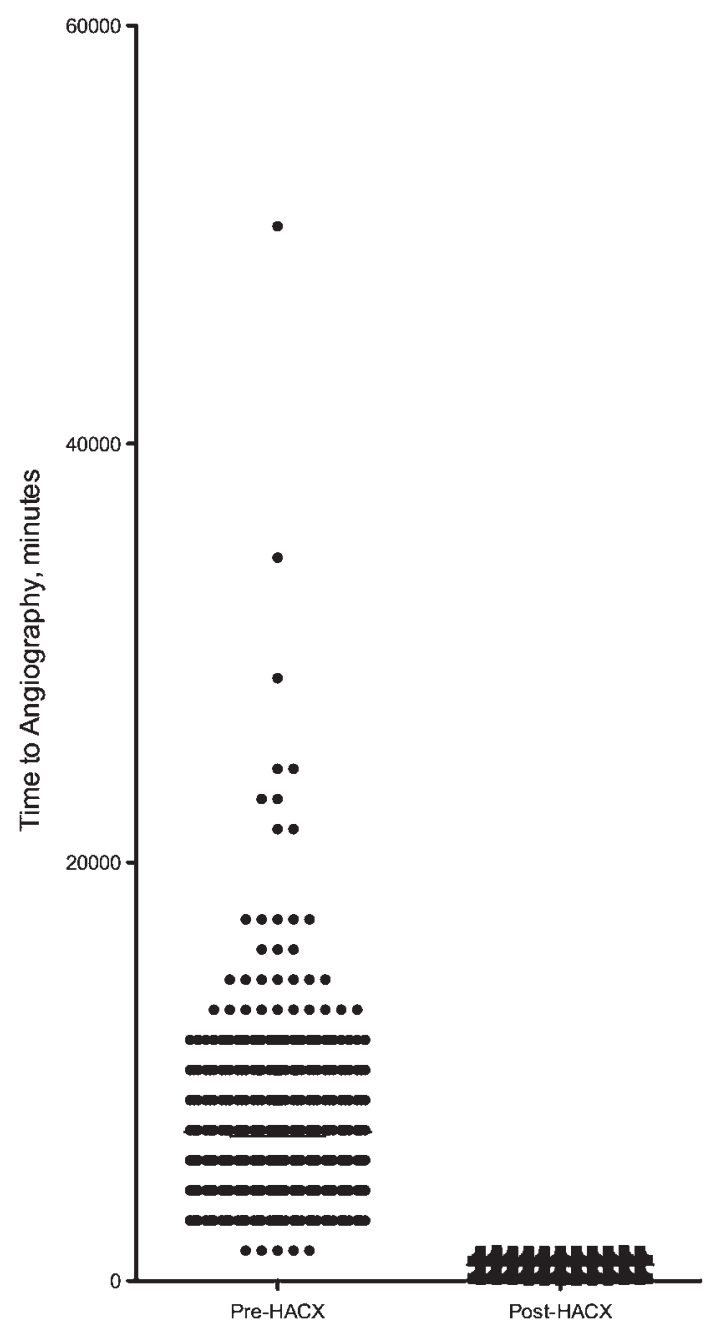

Abstract 2 Figure 1 Time from ED presentation to coronary angiography. wait for transfer to tertiary centre was $4.1( \pm 4.7)$ days. Median length of stay for HACX patients was 3 days. HAC-X has reduced wait for coronary angiography by 3.4 days per patient.

Conclusions This novel care pathway allows delivery of early angiography to NSTEMI patients in accordance with national guidance. Importantly, the pathway allows accurate diagnosis of NSTEMI, and inappropriate transfers are infrequent. Its introduction has resulted in a significant reduction in time to angiography for NSTEMI patients, and significant reductions in total hospital bed occupancy for patients with NSTEMI.

\section{SURVIVAL FOLLOWING ACUTE MYOCARDIAL INFARCTION IN PATIENTS OF SOUTH ASIAN AND WHITE EUROPEAN ETHNICITY IN THE UK}

doi:10.1136/heartjnl-2011-300198.3

${ }^{1} \mathrm{~N} N$ Gholap, ${ }^{2} \mathrm{R}$ L Mehta, ${ }^{2} \mathrm{~K}$ Khunti, ${ }^{2} \mathrm{M}$ J Davies, ${ }^{2} \mathrm{~B}$ Squire. ${ }^{1}$ University Hospitals of Leicester, Leicester, UK; ${ }^{2}$ University of Leicester, Leicester, UK

Introduction Some UK studies have suggested higher case-fatality rates following acute myocardial infarction (AMI) in British South Asian (SA), compared to white European (WE) people, driven by higher prevalence of diabetes in the SA ethnic group. However other studies have suggested similar or even better adjusted overall post-AMI survival for these ethnic groups. In patients with AMI, both prior diagnosis of diabetes as well as acutely elevated blood glucose regardless of diabetes status are associated with adverse outcomes. The aim of this study was to compare survival rates following AMI in SA and WE patients drawn from a contemporary, multi-ethnic UK population. Methods: We conducted a retrospective cohort study of total 4111 (SA 18\%) consecutive patients with AMI admitted between October 2002 and September 2008. Baseline differences between the ethnic subgroups were examined using independent two-sample $t$ tests for continuous and $\chi^{2}$ tests for categorical variables. Cox regression models were constructed to identify determinants of 30-days and 1year mortality, entering ethnicity, random admission blood glucose and antecedent diabetes individually and together along with other relevant variables.

Results: SA patients were younger (62 vs 67 years, $p<0.005$ ) and less likely to have smoked ( $16 \%$ vs $40 \%, \mathrm{p}<0.005)$ but more likely to have hypertension ( $55 \%$ vs $49 \%, \mathrm{p}=0.004$ ) or diabetes ( $40 \%$ vs $16 \%$, $\mathrm{p}<0.005)$ at presentation compared to WE patients. All cause 30 -day and 1-year mortality proportions were $10.0 \%$ and $15.2 \%$ in SA compared to respectively $9.9 \%$ and $16.7 \%$ in WE patients. For SA ethnicity, the univariate HR of 30-day mortality was 1.01 (95\% CI 0.79 to 1.30$)$ compared to WE ethnicity. On multivariate analysis (excluding antecedent diabetes and admission blood glucose) this association of SA ethnicity and mortality became significant (HR 1.56, CI 1.10 to 2.23) and remained so when antecedent diabetes was added to the analysis (HR 1.48, CI 1.03 to 2.13). However when admission blood glucose was added to the model, association of ethnicity with mortality became non-significant (HR 1.31, CI 0.86 to 1.99). Conversely each unit ( $\mathrm{mmol} / \mathrm{l})$ increase in admission blood glucose was associated with $7 \%$ increase in mortality (HR 1.07, CI 1.04 to 1.10) in this model, after adjusting for all the covariates. Furthermore exclusion of ethnicity and antecedent diabetes from the model did not alter the predictive value of admission blood glucose (HR 1.08, CI 1.05 to 1.10). Similar associations were observed for 1-year mortality.

Conclusions Despite higher prevalence of diabetes in SA patients, their mortality post AMI was similar to WE patients. Furthermore, admission hyperglycaemia more so than antecedent diabetes was an important predictor of increased mortality post AMI. To improve survival, active management of admission hyperglycaemia should be considered in patients admitted with AMI, regardless of their diabetes status or ethnicity. 